\title{
The Effect of EDTA Chelation Therapy in Symptomatic Coronary Heart Disease: An Observational Study
}

\author{
Chulananda D. A. Goonasekera ${ }^{1}$, Rohini Tennakoon ${ }^{2}$, Premil N. Rajakrishna ${ }^{3}$, Gammadegedara A. \\ Gunasena $^{3}$, Chandima R. Wanniarachchi ${ }^{3}$, Asanka B. Yatawatta ${ }^{3}$, Udawatta A. D. D. Munidasa ${ }^{3}$ \\ ${ }^{1}$ Department of Anesthesiology, Faculty of Medicine, Peradeniya \\ ${ }^{2}$ Consultant Cardiologist, General Hospital, Kandy, Peradeniya \\ ${ }^{3}$ Temporary Lecturer, Department of Anesthesiology, Faculty of Medicine, Peradeniya \\ E-mail: cgoonase@slt.lk \\ Received May 7, 2010; revised July 8, 2010; accepted July 26, 2010
}

\begin{abstract}
Ethylene Diamine Tetra Acetic Acid (EDTA) chelation therapy has been considered a definitive alternative therapy for by-pass surgery in atherosclerotic cardiovascular disease for more than four decades. It is a relatively inexpensive method believed to restore blood flow in atherosclerotic vessels. However, the benefits of chelation therapy yet remain controversial in the treatment of ischemic heart disease. We observed the effect of EDTA chelation therapy on exercise tolerance in 13 volunteering patients receiving conventional treatment for established symptomatic coronary heart disease. Each patient received 30 weekly infusions of EDTA followed by monthly 12 boosters according to the ACAM protocol (American College for Advancement in Medicine). This was in addition to the conventional therapies they received from their respective physician in hospital. Stress ECG, echocardiography and coronary angiogram findings were obtained at the beginning of treatment. The distance that a patient could walk on level ground at moderate speed and the number of steps he/she can climb up on a staircase until he/she begins to feel either chest pain or breathlessness were the two clinical parameters of exercise tolerance recorded to grade angina. Liver and renal functions were tested at $1^{\text {st }}, 5^{\text {th }}, 10^{\text {th }}, 15^{\text {th }}$ and $30^{\text {th }}$ infusions. Of the 13 patients, 11 showed improvement in angina grading whilst 2 experienced no effect. One patient improved from angina grade IV to I, 6 from grade III to I, 1 from grade III to II and 3 from grade II to I. A statistically significant reduction in the mean score $(\mathrm{p}=$ 0.002) was noticed at $6^{\text {th }}$ month of treatment when compared to that of the first month. A significant 1.7 fold increase $(p=0.009)$ in the mean SGPT level was observed at the $30^{\text {th }}$ infusion when compared to the pre-treatment values. The SGOT level showed no significant change $(p=0.664)$. None of the patients showed clinical features of hepato-cellular damage. The mean serum creatinine level showed a trend for reduction ( $p=0.083$ ) with treatment. The recognized side effects of intravenous EDTA chelation therapy such as liver damage, renal damage, hypersensitivity, symptomatic hypocalcaemia, and thrombophlebitis were not encountered. Thus, EDTA chelation therapy as prescribed by the ACAM protocol seems safe and effective in improving exercise tolerance in ischemic heart disease when administered concurrently with conventional therapy.
\end{abstract}

Keywords: EDTA Chelation Therapy, Coronary Heart Disease, Exercise Tolerance

\section{Introduction}

Ischaemic heart disease (IHD) resulting from the narrowing of coronary arteries due to atheromatous plaques, is the commonest cause of deaths in Sri Lanka [1]. Its definitive treatment comprise of revascularization surgery [i.e. Percutaneous Transluminal Coronary Angio- plasty (PTCA), or Coronary Artery Bypass Grafting (CABG)]. Its symptomatic management includes drug therapy (aspirin, beta blockers, nitrates, etc.) and lifestyle modifications. Surgical revascularization procedures are costly and not widely available. Often, most needy patients are unsuitable for surgery due to their poor physical condition or age. In this context, the search for an 
alternative, definitive and cost effective treatment is important. One such treatment is "chelation therapy" i.e. a therapy of intravenous Ethylene Diamine Tetra Acetic Acid (EDTA), a synthetic amino acid.

The benefits of EDTA Chelation therapy practiced worldwide since 1950s are still unclear. This is because of scarcity of well-controlled clinical trials. The exact mechanism of action of EDTA is not yet fully understood. One theory suggests that EDTA might work by removing calcium from atheromatous plaques clearing the blocked coronary arteries. Others believe that EDTA reduces oxidative stress related damage on coronary vessels [2]. Some evidence suggests that EDTA reduces platelet aggregation [3].

In 2001, a metanalysis of 19 studies with data on 22765 patients revealed a statistical correlation coefficient of 0.88 , which was indicative of a positive relationship between EDTA therapy and improved cardiovascular function [4]. There were also a few studies such as the Calgary PATCH EDTA chelation therapy study 2002, which stated that there was no evidence to support chelation therapy in patients with ischaemic heart disease, stable angina, and positive treadmill test for ischemia [5]. This study has been criticized as non scientific [6].

In spite of above controversies, "Chelation Therapy" for ischaemic heart disease is practiced in many countries including USA, UK, Australia and New Zealand using accepted protocols (ACAM, Rozema TC). An overview of all clinical investigations of chelation therapy conducted in the year 2000 states that out of 22 uncontrolled studies, 20 reported subjective symptomatic improvement [7]. A study of 18 patients using Technetium 99m echocardiography has shown a statistically significant improvement in left ventricular ejection fraction with chelation therapy [8]. Several recent reviews conducted on the subject, whilst acknowledging the fact that there is a scarcity of solid data to approve its use [9], conclude that EDTA therapy may be used in the context of a research trial [10] or as a 'last resort' cardiovascular revitalization strategy extending beyond revascularization [11] especially in patients considered unsuitable for revascularization [12]. On the other hand EDTA therapy as prescribed is not considered a highly invasive or harmful therapy [13]. As a result groups of physicians in Sri Lanka conduct chelation therapy as an 'alternative medicine' for CABG in ischaemic heart disease, on a non-profit basis, for volunteering patients.

This paper presents an observational study of outcomes, adverse effects, exercise tolerance and hepato renal function in 13 such patients who have completed a standard course of EDTA chelation therapy over the past three years at a non-profit clinic conducted in the Central
Province of Sri Lanka.

\section{Methods}

Volunteering patients, self referring themselves for chelation therapy whilst receiving conventional drug treatment for established ischaemic heart disease qualified for recruitment for this study. Subjects with co-morbid conditions such as a) psychiatric illness affecting understanding b) severe renal impairment (s. creatinine > $2.5 \mathrm{mg} / \mathrm{dl}$ ) c) significant liver disease (liver enzymes> twice the upper limit) d) severe cardiac failure e) allergy to EDTA f) suspected lead encephalopathy or else if pregnant were excluded.

Informed written consent was obtained after explaining the background, the ambiguous nature of treatment, and side effects. Their pre-treatment assessment included a history, physical examination and baseline investigations: i.e. urine for sugar and protein, serum creatinine, full blood count, SGOT, SGPT, lipid profile, fasting blood sugar, ECG, 2D Echocardiography, and treadmill exercise test (modified Bruce Protocol). All angina patients were graded according to the Canadian Cardiovascular Society Classification System before the treatment, namely, Class I-No limitation of physical activity (Ordinary physical activity does not cause symptoms.), Class II-Slight limitation of physical activity (Ordinary physical activity does cause symptoms.), Class IIIModerate limitation of activity (Patient is comfortable at rest, but less than ordinary activities cause symptoms.), and Class IV-Unable to perform any physical activity without discomfort, therefore severe limitation (Patient may be symptomatic even at rest) [14].

ACAM Protocol for the safe and effective administration of EDTA Chelation therapy was followed [8]. Each patient was given weekly 30 infusions followed by monthly 12 boosters. The duration of therapy was approximately $1 \frac{1}{2}$ years. The therapeutic infusions were given over a period of 3 hours according to the following formula.

The EDTA dose, rate of infusion and the frequency of administration were re-adjusted according to the creatinine clearance. If the creatinine clearance was $100 \mathrm{ml} /$ $\mathrm{min} / 1.73 \mathrm{~m}^{2}$, full dose of EDTA was given. If it was below $100 \mathrm{ml} / \mathrm{min}$, for example $70 \mathrm{ml} / \mathrm{min} / 1.73 \mathrm{~m}^{2}$, then $70 \%$ of the EDTA dose was given. The total fluid load was reduced to $400 \mathrm{ml}$ or $300 \mathrm{ml}$ in patients presenting with clinical features of heart failure (lung basal crepitations, ankle oedema, etc.) [8].

Patients were advised to take an adequate meal prior to the infusion. The infusion was given in a comfortable sitting position and patients were served with refreshments and drinking water if required. 
Investigations were repeated soon after the $5^{\text {th }}, 10^{\text {th }}$, $15^{\text {th }}$ and $30^{\text {th }}$ weekly infusions to assess their renal and liver functions. Their lipid profiles were checked at $10^{\text {th }}$ and $30^{\text {th }}$ infusions. The distance that a patient could walk on level ground at moderate speed and the number of steps he/she can climb up on a staircase until he/she begins to feel either chest pain or breathlessness were recorded prior to, and at the end of the first course (30 infusions) of EDTA therapy.

The importance of life style adjustment measures was emphasized. Regular aerobic exercise, quitting smoking and alcohol, stress free life, and dietary control measures such as limitation of salt, sugar, and fat intake and increase in fiber content were the key advices conveyed to the patients via leaflets and interviews.

Angina grading was reassessed at the end of 30 weekly infusions using two subjective parameters; namely a) the distance that a patient could walk on level ground at moderate speed and $b$ ) the number of steps he could climb up on a staircase until he begins to feel either chest pain or breathlessness. The patient's opinion on the effectiveness of the treatment was also sought. The biochemical parameters; Urine sugar and protein, Serum creatinine, Full blood count, and SGOT, SGPT, and Lipid profile were repeated.

All causes of death, myocardial infarction, stroke, hospitalization for angina, and coronary revascularization surgery were taken as study end points.

\section{Results}

All 13 patients (10 male, age median 57yrs, range 45-71) completed 30 infusions. Of the 13, 10 patients had had coronary angiograms and 9 had been recommended to have CABG. Their angina grading before and after treatment is shown in Table 1.

Of those 9 patients, 7 improved in angina grading. One patient recommended for medical management also improved.

Of 11 patients with stress ECGs, 7 were positive at stage I, 2 at stage II, and 2 at stage III. All patients improved in angina grading except for the 2 patients who were positive at stage I.

The mean blood pressures of our study group during treatment are shown in Figures 1 \& 2. The reduction in the mean systolic blood pressure at $3^{\text {rd }}, 4^{\text {th }}, 5^{\text {th }}$ and $6^{\text {th }}$ months were statistically significant when compared to that of the first month.

The patient's opinion on the effectiveness of treatment obtained after completion of the 30 infusions is shown in Table 2. The number of steps they could walk on flat ground and climb significantly improved as shown in Figure 3 and Figure 4 respectively.
Table 1. Angina grading before and after EDTA therapy course i.e. 30 infusions.

\begin{tabular}{ccc}
\hline \multicolumn{2}{c}{ Angina Grading } & N $=$ \\
\cline { 1 - 2 } Before infusions & After 30 infusions & \\
\hline IV & I & 1 \\
III & I & 6 \\
III & II & 1 \\
III & III & 1 \\
II & I & 3 \\
II & II & 1 \\
\hline
\end{tabular}

Table 2. Patients' perception on the effectives of the treatment.

\begin{tabular}{cc}
\hline Patients' opinion & Number of patients \\
\hline Symptoms totally improved & 2 \\
Symptoms improved to a greater extent & 7 \\
Symptoms improved to a lesser extent & 2 \\
No change & 2 \\
Symptoms worsened & 0 \\
\hline
\end{tabular}

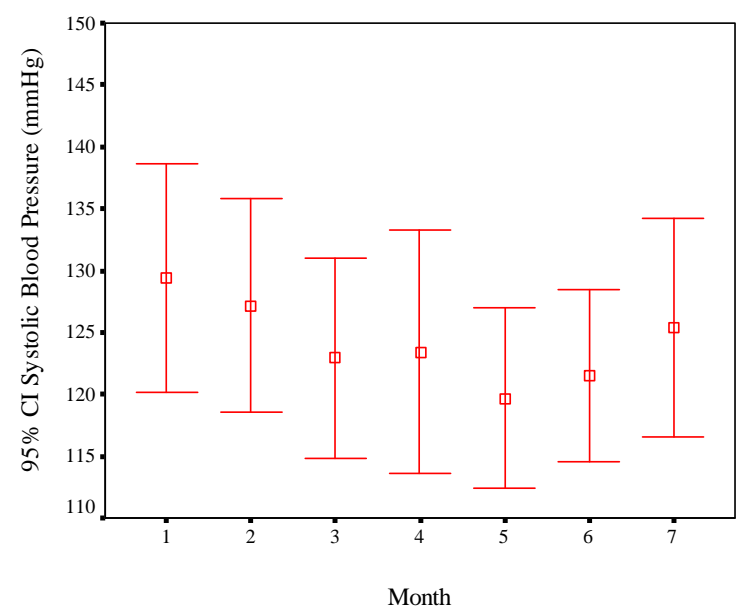

Figure 1. Monthly systolic blood pressure during chelation therapy.

The mean SGPT levels before and after treatment were $29.19 \mathrm{U} / \mathrm{L}$ and $41.58 \mathrm{U} / \mathrm{L}$ and showed a significant 1.42 fold rise $(p=0.009)$. The mean SGOT was $27.64 \mathrm{U} / \mathrm{L}$ and 32.64U/L showed no significant change $(\mathrm{p}=0.664)$. The mean serum creatinine level was $1.10 \mathrm{mg} / \mathrm{dl}$ before and $1.02 \mathrm{mg} / \mathrm{dl}$ after treatment and showed a declining trend $(\mathrm{p}=0.083)$.

Major complications of EDTA i.e. liver damage, renal damage, hypersensitivity, symptomatic hypocalcaemia and thrombophlebitis were not seen in our patients. One patient developed faintishness after her $2^{\text {nd }}$ infusion. Another patient quit the treatment at the $5^{\text {th }}$ infusion, complaining that a rash in the leg exacerbated with the infusion. 


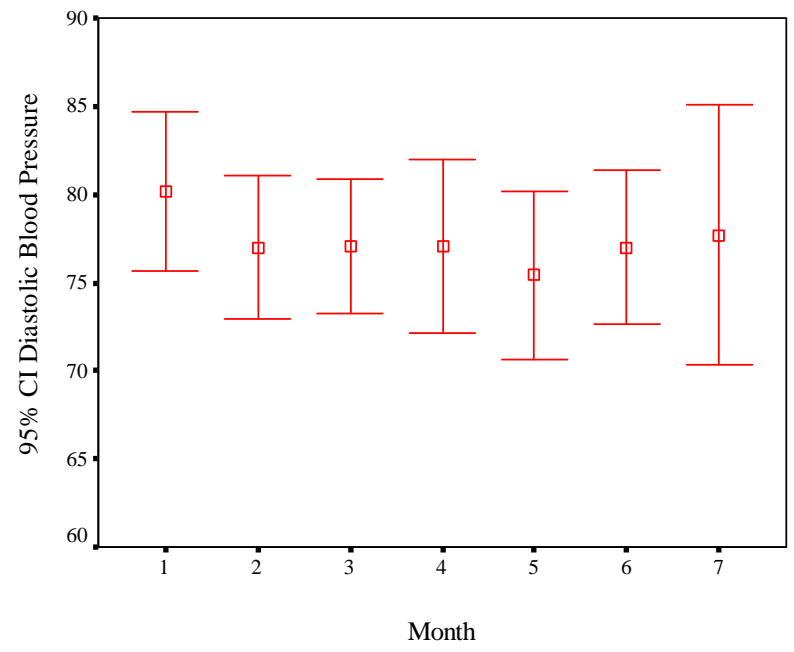

Figure 2. Monthly diastolic blood pressure during chelation therapy.

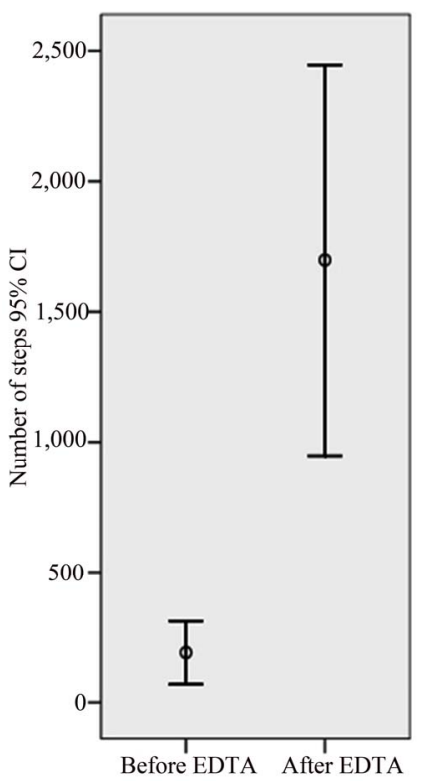

Figure 3. The number of steps subjects could walk on flat ground before and after the completion of full course of EDTA.

\section{Discussion}

We studied the effect of EDTA therapy in 13 patients with established symptomatic ischaemic heart disease who were on conventional drug treatment with life style modifications. They were also either considered not suitable for cardiac revascularization or were not willing to undergo the procedure. Of the 13 patients, 11 showed an improvement in angina grading at least by one class.

Their exercise tolerance as indicated by walking distance and climbing ability substantially improved. Seven

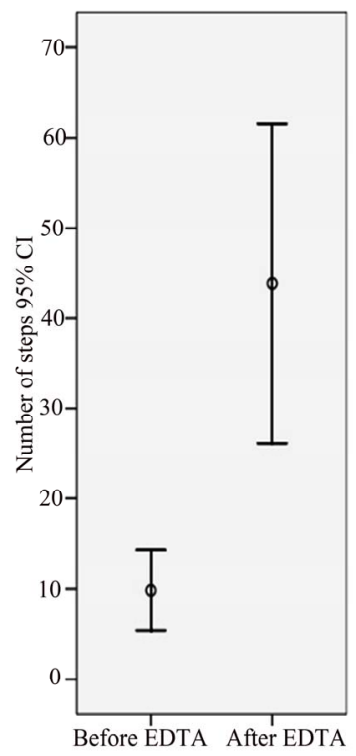

Figure 4. The number of steps subjects could climb before and after the completion of full course of EDTA

patients who had been recommended for early CABG were within the improved group.

Objective parameters to assess the effect of EDTA, such as stress ECG for exercise tolerance, echocardiography for cardiac function were not used in this study as patients could not afford the expenses.

The mean blood pressure of our study group was within the normal range. Interestingly we found a significant reduction in systolic blood pressure between $8^{\text {th }}$ and $24^{\text {th }}$ week of EDTA infusion therapy. Most of these patients were on antihypertensive medication and regular physical exercise, which are known to control blood pressure. It has also been previously reported that chelation therapy lowered the systolic blood pressure at all stages of the sub maximal treadmill stress test in both exercising and non exercising groups [15]. Therefore, it is worth considering further research to elucidate whether chelation therapy has a permissive action on controlling hypertension.

Critics of chelation therapy have frequently suggested that reported improvements are a placebo effect. A placebo effect begins shortly after its first administration and rarely, persists for more than three months. Benefits shown in our study are 30 weeks apart. Chelation therapy, by contrast, shows its full range of benefits quite slowly. Usually it required not only several months of therapy but also an additional several months after the course of therapy for the full benefit of treatment to occur. The benefits accrued generally persist for years thereafter [16].

The results we obtained might have been affected by other confounding factors such as vitamin B supplemen- 
tation, life style modifications and conventional drug therapy. Several studies have shown that favorable life style modifications like quitting smoking, regular exercise and dietary adjustments have a significant effect on attenuating abnormal cardiovascular risk factors [17].

Some authors continue to refuse EDTA Chelation therapy claiming that it has serious side effects including renal and hepatic toxicity. EDTA does have them when given at higher doses for heavy metal poisoning. So would it be for any other medication if toxic doses are used [2]. However, with the use of accepted protocols (e.g. ACAM) for EDTA therapy in heart disease none of the major side effects have been reported in previous studies. Out observations are similar.

SGPT levels were within the normal range before treatment and only two patients had values slightly above the upper normal at the end of treatment $(46.4 \mathrm{U} / \mathrm{L}$ and $57 \mathrm{U} / \mathrm{L}$; normal range 0-40 U/L). One patient who had elevated SGOT level at the end of treatment (99 U/L) also had an abnormal level (47 U/L) pre-EDTA treatment. However these post treatment liver enzyme elevations are relatively low when compared to that of known liver pathologies [18]. Since the study group was on statins (a group of cholesterol lowering drugs known to raise liver enzymes) as a component of their conventional therapy, a reliable causal relationship between EDTA and raised liver enzymes could not be made. However, none of our patients showed any clinical features of hepato-cellular damage during the study period.

We observed a decreasing trend in the mean serum creatinine level during treatment. EDTA induced renal damage is a very rare adverse effect and it is usually associated with higher doses [19]. There is even a suggestion that this treatment procedure may improve renal function. A study carried out on 383 subjects with chronic degenerative disorders who were treated with EDTA for 50 days showed an overall decline in fasting serum creatinine levels [20].

Other known side effects of intravenous EDTA chelation therapy (hypersensitivity, symptomatic hypocalcaemia and thrombophlebitis) were not encountered during our study period. Intravenous access related complications were minor and rarely observed. Pain at the injection site and allergy to plastic tourniquet (mild erythema) troubled a rare patient.

\section{Conclusions}

We observe that EDTA chelation therapy is effective in improving the clinical outcome in a majority of IHD patients with negligible side effects when given according to approved protocols. Further research is needed to confirm this causal relationship. The variability of the effect of EDTA on different patients creates the need for further research to recognize the clinical subset of IHD patients for whom it is most suitable.

\section{Acknowledgements}

We thank all staff members of EDTA chelation therapy clinic, Kandy, and all patients who participated. We appreciate the support received from the Torance Company Pharmaceuticals to import the relevant drugs under a special license.

\section{References}

[1] World Health Organization, "Mortality Country Fact Sheet," 2006. http://www.who.int/whosis/mort/profiles/mort_searo_ lka_srilanka.pdf

[2] NCCAM, National Institutes of Health, "Questions and Answers: The NIH Trial of EDTA Chelation Therapy for Coronary Artery Disease,” 2004. http://nccam.nih.gov/ health/chelation/q-and-a.htm

[3] G. Kindness and J. P. Frackleton, "Effect of Ethylene Diamine Tetra Acetic Acid (EDTA) and Platelet Aggregation in Human Blood," Journal of Advancement in Medicine, Vol. 2, No. 4, 1989, pp. 519-530.

[4] L. Chappell and J. P. Stahl, "The Correlation between EDTA Chelation Therapy and Improvement in Cardiovascular Function: A Meta Analysis,” Journal of Advancement in Medicine, Vol. 6, No. 3, 1993, pp. 139-160.

[5] M. L. Knudtson, D. G. Wyse, P. D. Galbraith, R. Brant, K. Hildebrand, D. Paterson and D. Richardson, "Chelation Therapy for Ischemic Heart Disease: A Randomized Controlled Trial," Journal of the American Medical Association, Vol. 287, No. 4, 23-30 January 2002, pp. 481486.

[6] “Critique of the Calgary PATCH EDTA Chelation Study," Adapted from a report by Croft Woodruff in Canada, 2007. http://www.drcranton.com/calgarystudy.htm

[7] E. Ernst, "Chelation Therapy for Coronary Heart Disease: An Overview of All Clinical Investigations," American Heart Journal, Vol. 140, No. 1, July 2000, pp. 139-141.

[8] E. M. Cranton, "Protocol of the American College of Advancement in Medicine for the Safe and Effective Administration of EDTA Chelation Therapy". Journal of Advancement in Medicine, Vol. 2, No. 1-2, 1989, pp 269305.

[9] E. Ernst, “Chelation Therapy for Coronary Heart Disease: An Overview of All Clinical Investigations," American Heart Journal, Vol. 140, No. 1, July 2000, pp. 139-141.

[10] J. S. Shrihari, A. Roy, D. Prabhakaran and K. S. Reddy, "Role of EDTA Chelation Therapy in Cardiovascular Diseases," National Medical Journal of India, Vol. 19, No. 1, January-February 2006, pp. 24-26.

[11] P. M. Kidd, "Integrative Cardiac Revitalization: Bypass Surgery, Angioplasty, and Chelation. Benefits, Risks, and Limitations," Alternative Medicine Review, Vol. 3, No. 1, 
February 1998, pp. 4-17.

[12] H. Quan, W. A. Ghali, M. J. Verhoef, C. M. Norris, P. D. Galbraith and M. L. Knudtson, "Use of Chelation Therapy after Coronary Angiography,” American Journal of Medicine, Vol. 111, No. 9, 15 December 2001, pp. 686691.

[13] D. M. Seely, P. Wu and E. J. Mills, "EDTA Chelation Therapy for Cardiovascular Disease: A Systematic Review," BMC Cardiovascular Disorders, Vol. 5, 2005, p. 32.

[14] L. Campeau, “Grading of Angina Pectoris,” Circulation, Vol. 54, No. 3, September 1976, pp. 522-523.

[15] E. W. McDonagh, C. J. Rudolph, E. Cheraskin and D. G. Wussow, "The Effect of EDTA Chelation and Supportive Multivitamin/Trace Mineral Supplementation with and without Physical Activity upon Systolic Blood Pressure” Journal of Orthomolecular Psychiatry, Vol. 13, 1984, pp. 1-9.

[16] E. M. Cranton and J. P. Frackelton, "Current Status of
EDTA Chelation Therapy in Occlusive Arterial Disease," 2001. http://www.drcranton.com/chelation/emcjpf.htm

[17] R. Yamamoto, T. Kawamura, K. Wakai, Y. Ichihara, T. Anno and Y. Mizuno, "Favorable Life-Style Modification and Attenuation of Cardiovascular Risk Factors," Japanese Circulation Society, Vol. 63, No. 3, March 1999, pp. 184-188.

[18] "EDTA Chelation: The Real 'Miracle' Therapy for Vascular Diseases,” Journal of Life Enhancement, 1997. http:// www.life-enhancement.com/article_template.asp?ID=78

[19] H. R. Casdorph, "EDTA Chelation Therapy, Efficacy in Arteriosclerotic Heart Disease,” Journal of Holistic Medicine, Vol. 3, No. 1, 1981, pp. 53-59.

[20] M. Elmer and E. M. Cranton, "Protocol of the American College for Advancement in Medicine for the Safe and Effective Administration of EDTA Chelation Therapy," Journal of Advancement in Medicine, Vol. 2, No. 1-2, 1989, pp. 269-303. 